\title{
THE RADIOLOGICAL MANAGEMENT OF TUBERCULOSIS
}

\section{*O Ogbeide}

*Department of Radiology, University of Benin Teaching Hospital,

Benin City, Nigeria

\section{Correspondence:}

Dr Anthony .O Ogbeide

Department of Radiology,

University of Benin Teaching Hospital,

P.M.B. 1111 Ugbowo

Benin City, Nigeria

Email: tonyosagbeide@yahoo.com

\section{INTRODUCTION}

Tuberculosis is a common multisystemic infectious disease caused by mycobacterium specie mainly mycobacterium tuberculosis. It affects everyone from Infants to the very aged.

Tuberculosis usually affects the lungs (pulmonary tuberculosis) in $75 \%$ of cases, but in $25 \%$ of cases the other body systems: Central nervous system, lymphatic system, genitourinary system, gastrointestinal system, bones and joints may be involved collectively denoted as extrapulmonary tuberculosis.

In the radiological management of tuberculosis (TB), imaging modalities that are utilized cover both pulmonary and extra-pulmonary diseases. The modality of choice, however, depends on the clinical complains of an individual as all may not apply to a single individual patient. Imaging modalities that may be employed include-Plain-x-ray examinations, Computed tomography, Ultrasound scan, Magnetic resonance imaging Radionuclide studies, Intravenous urography, Barium studies and Hysterosalpingography.

\section{ROLE OF IMAGING}

The management of tuberculosis may require a multi-disciplinary approach depending on the organ system involved in the disease process, and radiology plays a very significant role in its management. The role of imaging thus includes the following:

------Detection of TB

------Determination of disease activity

------Detection of complications

------Determination of response to therapy

------Providing roadmap for surgical team planning

------Interventional procedures

\section{PATTERNS OF IMAGING FINDINGS Pulmonary manifestation}

Chest $\mathrm{x}$-ray is the mainstay in the radiological evaluation of suspected or proven pulmonary TB. Computed tomography may be useful in clarifying confusing findings and could better delineate lesions. The findings in these modalities may suggest whether the disease is primary or post-primary TB; and the radiological features are closely related to the level of host immunity ${ }^{1}$.

Children and the immunocompromised persons usually present with features of primary TB which are highlighted thus:

Parenchymal infiltrates or consolidation------opacification of airspaces within the lung parenchyma, involving any pulmonary lobe or segment (Fig: I). 
Fig: I

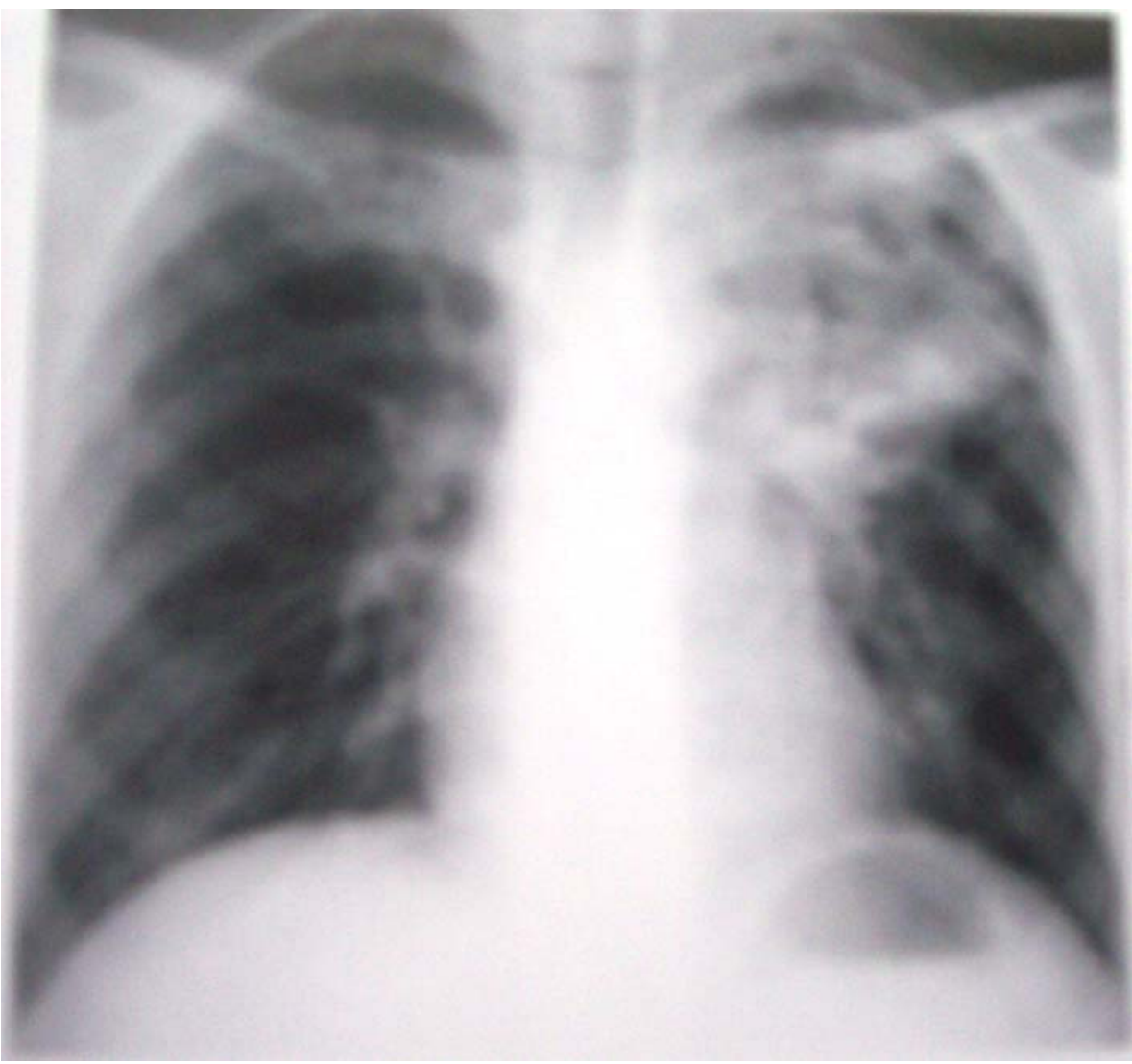

Any of these patterns may appear on the first chest radiograph, separately or in any combination. In patients with poor immunity for any reason, the primary infection may never heal but continue on to severe cavitating spreading pneumonia. This is progressive primary tuberculosis. The majority of primary infection, however, heal with little residual scaring; only a few will imperceptibly change to the secondary or immune pattern of tuberculosis with resulting fibrosis, distortion and calcification ${ }^{2}$.

Post-primary TB typically manifest as a heterogenous consolidation (opacity) often with cystic or cavitory changes usually in the apical and posterior segments of the upper lobes and the superior segment of the lower lobes (Fig: IV). Cavitations (marginated lucencies) are the most important radiological findings in postprimary disease. Lymphadenopathy is rare. There may be nodular and or fibrotic (reticular) densities in the lung parenchyma. These features may be associated with features of volume loss which may include---displacement of the interlobar fissures, opacification of the involved lung, hilar displacement, mediastinal displacement, elevation of the diaphragm, rib crowding and compensatory emphysema ${ }^{3}$.

Pulmonary TB may be complicated by features of bronchiectasisbronchial dilatation with bronchial wall thickening.

Activity of post-primary disease cannot be accurately assessed by chest radiography. Radiographic stability for 6 months and negative 
sputum cultures is the best indicator of inactive disease.

Hilar or mediastinal lymphadenopathy with or without associated atelectasis (Fig: II)

Tuberculous pleurisy manifesting as pleural effusion

Fig: II

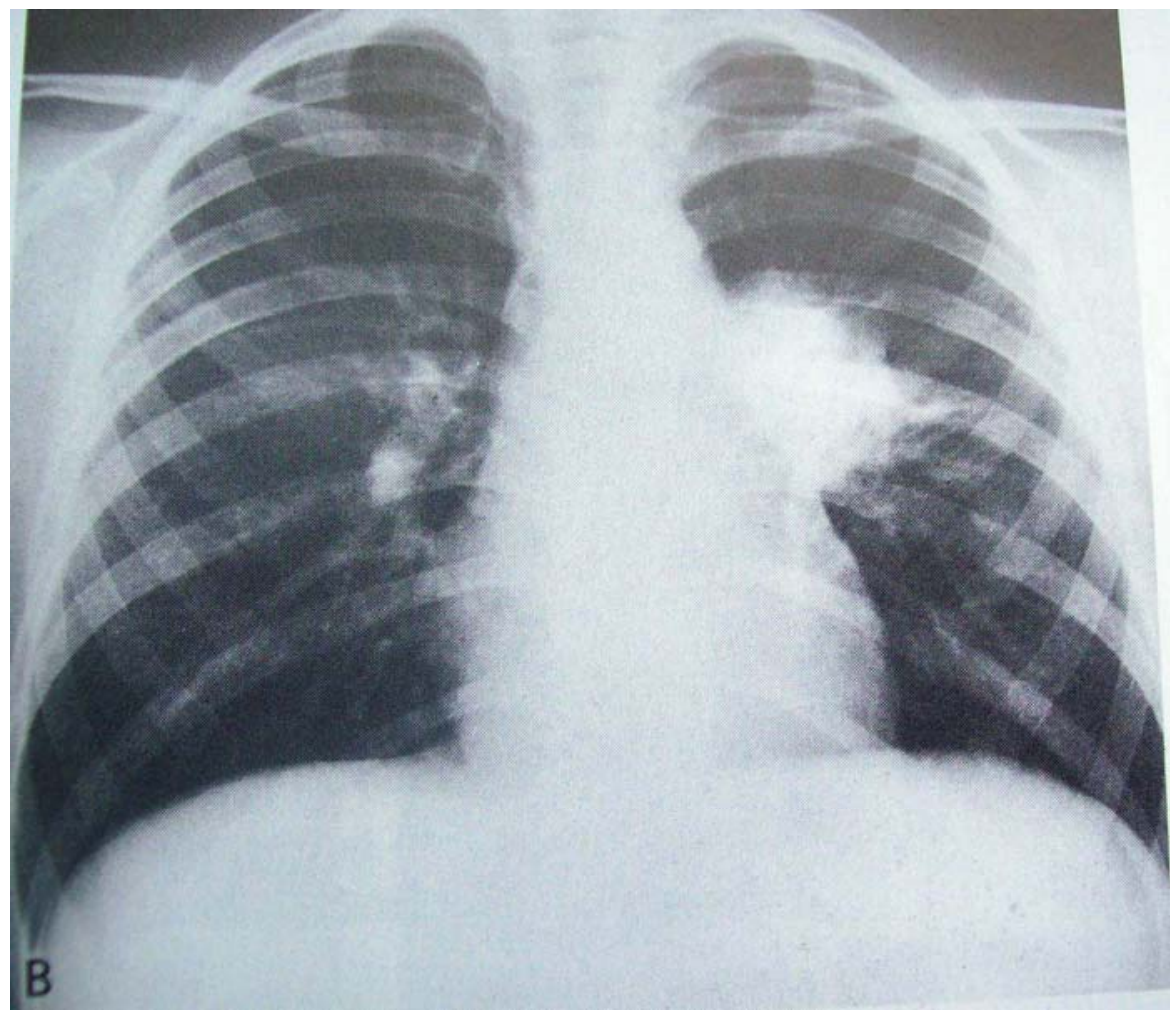

\section{Extra-pulmonary manifestation}

The extra-pulmonary manifestation of tuberculosis cuts across the various body system and as such the radiological evaluation of these sites employ imaging modalities that best define the suspected lesions at the specific organ sites. For example, ultrasonography and intravenous urography may be useful in the evaluation of the renal system while hysterosalpingography in addition to ultrasound scan may be used to assess the female reproductive system. Plain $\mathrm{x}$-ray examinations are of relevance in imaging the skeletal system while barium meal/enema are used to evaluate the gastrointestinal tract. However, the features seen on the modalities are not specific for tuberculosis as other disease conditions may simulate similar features. Some of the various manifestations that may be seen at these sites are stated thus:

Skeletal tuberculosis----arthritis, collapsed vertebra, paraspinal masses, kyphosis, granulomas Central nervous system tuberculosis---tuberculoma, abscess, meningitis (with or without hydrocephalus) Gastrointestinal tuberculosis---lymphadenopathy, stricture, dilatation Genitourinary tuberculosis---granuloma, calcification, cavitation, strictures, oedema, ulceration, abscess. 
Miliary disease----nodular opacities of millet-size $(1-2 \mathrm{~mm})$ distributed through out the lung parenchyma.

Destroyed lung syndrome (Fig: III)

Fig: III

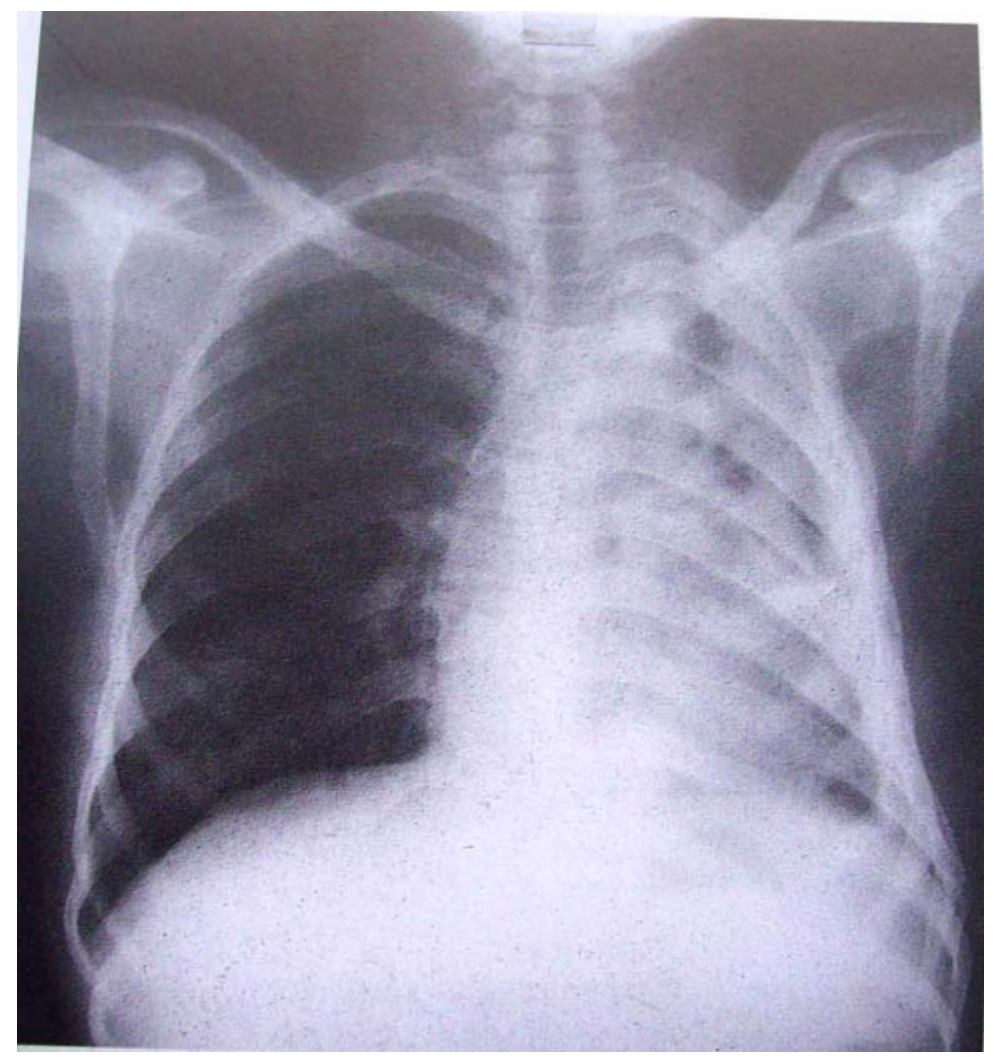

\section{THE VALUE OF RADIONUCLIDE STUDIES}

Radionuclide scanning may also play a role in the management of TB. Although it is not specific for TB it however has some merits.

Positron-emission-tomography (PET) scan using Fluorine-18 FDG or C-Choline can sometimes help differentiate tuberculous granuloma from lung malignancy ${ }^{4}$.

Bone scan using tecnitium-99 metastable may also be useful in delineating and localizing sites of active lesions and these are seen as areas of increased uptake (photonrich) but this feature is not pathognomonic of TB.

\section{INTERVENTIONAL MANAGEMENT} Ultrasound scan and computed tomography beside being of diagnostic values may also be useful as interventional procedures for guided aspiration and guided needle biopsy for histopathological diagnosis. 


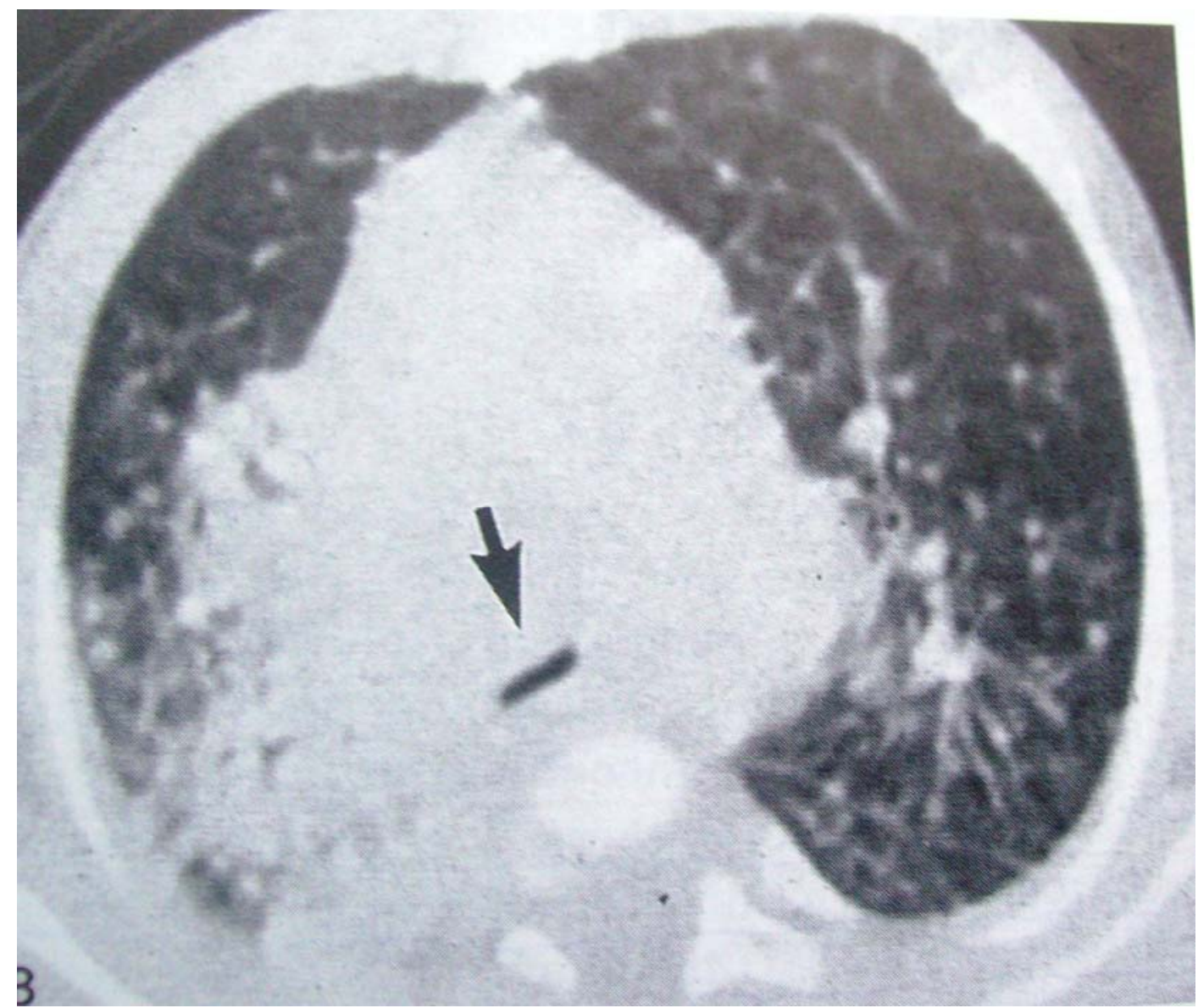

Fig: IV

\section{CONCLUSION}

In the overall management of patients with tuberculosis, early radiological evaluation is important to make prompt diagnosis and monitor the disease process in terms of response to drug treatment or otherwise to assess the extent of possible complications.

\section{REFERENCES}

1. Awil PO, Bowlin SJ, Daniel TM. Radiology of pulmonary tuberculosis and HIV infection in Gulu, Uganda. Eur Respr J.1997; 10: 615-618

2. Palmer PES, Reeder MM. The imaging of tropical diseases with pathological correlations. $2^{\text {nd }} \quad \operatorname{Rev}$ (ed.) 2001:397-398.
3. Sutton D, The textbook of Radiology and Imaging. $7^{\text {th }}$ edition. 2007:140-144.

4. Jeong YJ, Lee KS. Pulmonary Tuberculosis and up to date imaging and management. American Journal of Roentgenology 2008; 191:834844. 